Linguistique, littérature, didactique

\title{
Pour une formation linguistique aux écrits professionnels
}

\section{Fanny Rinck et Frédérique Sitri}

\section{(2) OpenEdition}

\section{Journals}

Édition électronique

URL : http://journals.openedition.org/pratiques/1937

DOI : $10.4000 /$ pratiques. 1937

ISSN : 2425-2042

Éditeur

Centre de recherche sur les médiations (CREM)

Édition imprimée

Date de publication : 15 juin 2012

Pagination : 71-83

Référence électronique

Fanny Rinck et Frédérique Sitri, «Pour une formation linguistique aux écrits professionnels », Pratiques [En ligne], 153-154 | 2012, mis en ligne le 16 juin 2014, consulté le 01 mai 2019. URL : http:// journals.openedition.org/pratiques/1937 ; DOI : 10.4000/pratiques.1937

(c) Tous droits réservés 


\title{
Pour une formation linguistique aux écrits professionnels
}

\author{
Fanny Rinck
}

Modyco, UMR7114, CNRS/Université Paris Ouest Nanterre La Défense

Frédérique Sitri

Syled/Cediscor, associée à Modyco, Université Paris Ouest Nanterre La Défense

\section{Introduction}

L'objectif de cet article est de s'interroger sur la conception et la mise en œuvre d'une formation du supérieur aux écrits professionnels. La maîtrise de ces écrits représente en effet une demande forte dans le monde de l'entreprise, demande qui se traduit par la multiplication des formations privées et des ouvrages sur la question. Notre parti pris est de considérer que la linguistique peut être mise à contribution pour bâtir ce type de formation, dans le sillage des travaux menés sur les genres universitaires et des propositions didactiques auxquels ils ont donné lieu.

Les recherches ont établi que dans tous les secteurs d'activité, les pratiques d'écriture sont de plus en plus diversifiées et exigent un niveau de maîtrise de plus en plus élevé pour tous (Barré De Miniac, 2003). L'écrit chez l'adulte est ainsi devenu un champ d'investigation (Bourgain, 1988 ; Dabène, 1987 ; Leclercq, 1999) et, avec la notion de littéracie (Barré et al., 2004 ; Wagner et al., 1999), la distinction entre ceux qui savent lire et écrire et ceux qui sont exclus de ces pratiques cède la place à une vision plus dynamique de l'écrit comme acculturation à des pratiques qui peuvent poser problème à tous, y compris à des diplômés de l'enseignement supérieur. 
Se pose alors la question du rôle de l'université dans la formation aux compétences rédactionnelles du monde professionnel. En quoi les pratiques d'écriture académique permettent-elles de former aux pratiques d'écriture professionnelles ? En quoi l'université peut-elle intégrer l'écrit professionnel dans les pratiques de formation? Si l'écrit professionnel suscite de l'intérêt dans le monde académique, c'est essentiellement pour l'heure à travers les écrits sur les pratiques comme outil de formation ${ }^{(1)}$. On pourrait ainsi qualifier ces derniers d'écrits professionnalisants et les distinguer des écrits professionnels en usage dans l'activité professionnelle, comme la lettre de motivation, la note de synthèse, le compte-rendu etc.

Les nombreux travaux menés ces dernières années sur le langage au travail et sur les écrits en usage dans des sphères d'activité spécifiques (par ex. Bourgain, 1988 ; Borzeix et al., 2001 ; Boutet et al., 2001 ; Delcambre, 1997 ; Filliettaz \& Bronckart, 2005 ; Alamargot et al., 2005, etc.), ou encore sur la rédaction technique (Klinkenberg, 2000) et la rédactologie (Beaudet, 2001), permettent d'affiner ces catégories d'écrits simplificatrices et d'identifier les genres, leurs enjeux et les difficultés des scripteurs, pour envisager alors des développements didactiques (par ex. Cislaru et al., 2007).

Confrontées à la mise en place d'une formation à la maîtrise rédactionnelle au sein d'un parcours de master dédié à l'écrit professionnel ${ }^{(2)}$, nous proposons de manière programmatique d'étudier en quoi les recherches en linguistique peuvent être mises à profit. L'enjeu est de dépasser le discours normatif sur les productions écrites ${ }^{(3)}$ en intégrant les apports de l'analyse de discours pour ne pas en rester à une vision techniciste et à des compétences rédactionnelles locales et décontextualisées. Dans un premier temps, le public visé n'est constitué que de «spécialistes » (futurs rédacteurs et formateurs à l'écrit), mais la démarche pourrait être réinvestie en partie dans le cadre d'un plan de formation à la maîtrise de l'écrit au niveau supérieur et/ou pour la formation continue de professionnels de secteurs spécifiques.

L'identification des besoins de formation s'appuie sur l'analyse de trois corpus, qui nous permet de montrer comment la linguistique peut apporter des réponses fondées en regard de ce que l'on sait sur la maîtrise de l'écrit. Les objectifs de formation que nous évoquons en conclusion ne constituent que de premières propositions et il nous semble important que la linguistique investisse ce champ de recherches, à la croisée des études sur les littéracies universitaires et sur le langage au travail. Les trois corpus utilisés se veulent des outils dans cette perspective : le premier porte sur les discours à visée de formation sur les écrits professionnels et en suggère les limites. Le second est un vaste chantier, celui de l'inventaire des genres en usage dans le monde professionnel. Le troisième se rattache aux corpus de productions d'étudiants et d'adultes, corpus

(1) En particulier dans la formation des enseignants, à travers les genres du rapport de stage et du mémoire professionnel (voir par ex. Crinon \& Guigue, 2006).

(2) Parcours Ecrifore (Ecriture, Formation, Remédiation) du Master Fonctionnements Linguistiques, Dysfonctionnements Langagiers, Université Paris Ouest Nanterre La Défense.

(3) Discours qui renvoie au «travail prescrit» plutôt qu'au «travail réel » (Clot, 1999). 
existants et à constituer pour l'analyse des besoins dans le cadre d'une formation du supérieur aux genres universitaires et professionnels.

\section{Les discours de formation sur les écrits professionnels}

Face au succès des manuels et formations de type " rédiger efficacement 》 chez les étudiants et les professionnels, il convient de s'interroger sur la vision de l'écrit et sur les normes qui circulent dans l'entreprise, ainsi que sur la pertinence linguistique des difficultés pointées et des aides rédactionnelles proposées.

Sur les sites et ouvrages que nous avons observés, ces discours utilisent massivement le vocabulaire de la communication. Il est question de manière récurrente de «communication efficace », de «performance », d' « utilité », de « fiabilité », de « clarté », de « rigueur». Ils proposent des « techniques » présentées comme transférables quelle que soit la situation (par exemple, la technique des «3C » (« être clair, concis, concret») ou l'idée d'un plan-type). Comme l'ont observé C. Oudart $(2001,2002)$ dans le champ de la formation d'adultes et V. Clavier et C. Laffont-Terranova (2005) dans les filières technologiques de l'enseignement supérieur, le «bien écrire » est envisagé comme une habileté générale, indépendante des spécificités des types d'écrits; quand ces dernières sont pointées, c'est sous la forme de modèles abstraits plutôt qu'en termes d'« outils scripturaux pertinents pour la situation de communication rencontrée » (Oudart, 2002).

La dimension linguistique intervient cependant à travers plusieurs entrées : au niveau pragmatique, avec l'accent mis sur le « destinataire » et la «fonction de 1'écrit », au niveau textuel (« mise en page », « plan et titres », " paragraphes » et « articulations logiques»), au niveau du « vocabulaire » (《trouver le mot juste », «éviter les répétitions ») et de la correction linguistique (« règles de grammaire », «fautes de français », « orthographe », "ponctuation »), et enfin à travers la notion de « style » (« varié », 《 concis », « dynamique »).

Une bonne partie des items envisagés et des recommandations qui leur sont associées recoupent les observations faites par D. Bourgain (1990) concernant les représentations de la norme écrite chez différentes catégories de professionnels (ouvriers, techniciens, administratifs, cadres) nonobstant les variations entre catégories : importance de l'orthographe, référence vague aux règles de grammaire, impératif de la phrase simple.

Cette dernière exigence va jusqu'à contredire les apports de la linguistique du texte, que nous évoquerons ici à travers une citation de M. Péri-Woodley (1993) à propos de la simplification syntaxique de textes scientifiques visant à les rendre plus lisibles par des adultes étudiant les sciences en anglais L2 :

« [Selon Lautamatti (1978a et b ; 1987)] loin de faciliter la lecture, cette simplification tend dans certains cas à la rendre plus difficile. Elle cite comme particulièrement problématique le découpage de phrases complexes en suites de 
phrases simples qui entraînent une multiplication de sous thèmes parmi lesquels le lecteur doit sélectionner les plus importants [...] la syntaxe complexe, par l'effet de relief qu'elle produit, est un procédé particulièrement important de structuration thématique. » (Pery-Woodley, 1993 : 74-75).

Nous ne reprenons ici que les grandes lignes d'un discours de formation dont on trouvera des analyses détaillées dans C. Oudart (2001) notamment. Les exigences qu'il met en avant reproduisent les représentations normatives que se font les scripteurs adultes de l'écrit et d'eux-mêmes (Dabène, 1987) et ne sont pas fondées en regard du fonctionnement de la langue et des mécanismes entrant dans la compréhension des textes. Ainsi, non seulement ces exigences ne donnent pas de réels outils de formation à l'écrit, mais elles peuvent s'avérer contreproductives en agissant comme obstacles pour le scripteur. Un travail sur le rapport à l'écriture des étudiants ou professionnels représente en ce sens une voie intéressante à travailler en formation, de même qu'un travail sur les compétences textuelles à même d'être mises au compte des critères de «qualité » que les travaux de description linguistique doivent permettre d'objectiver (Beaudet, 2001, 2002 ; Béguelin, 2000).

\section{Les genres en usage dans le monde professionnel}

Si les discours de formation visent une habileté générale de l'ordre de la « communication efficace » ou du « bien rédiger », certains mettent à l'honneur un ou quelques types d'écrits emblématiques du monde professionnel : lettres, rapports, synthèses etc.

Une analyse des écrits professionnels en termes de genres semble effectivement capitale compte-tenu de l'importance prise par la notion en linguistique et analyse de discours d'une part et en didactique de l'écrit et littéracies universitaires d'autre part. Cependant, le problème spécifique auquel on se heurte ici est celui d'une approche exploratoire visant à identifier les genres en usage dans de multiples sphères d'activité.

On sait que, si M. Bakhtine (1984) insiste sur le caractère innombrable et hétérogène des genres, il donne en fin de compte peu d'outils de classement. Or si le «nom de genre » constitue un indice non négligeable de l'évaluation par les utilisateurs de l'appartenance de tel texte à tel genre, on sait qu'il ne peut constituer le seul critère car « lorsqu' on adopte un point de vue descriptif et non plus normatif, on s'aperçoit qu'il n'y a plus recouvrement entre la définition sociale des genres (qui catégorise des individus prescrits dans des situations) et le point de vue formel (qui regroupe des productions langagières sur la base de marques linguistiques et de fonctionnements discursifs) »(Branca, 1999: 6).

Le champ de l'analyse de discours fournit ainsi des outils pour la constitution de corpus et pour une démarche d'identification des genres en relation avec les pratiques sociales où les textes sont en usage. A partir des propositions de D. Maingueneau (2004), S. Moirand (2003) et P. Von Munchow (2007), on proposera donc dans le cadre de la formation une approche du genre «multicritères » et «dynamique », prenant en compte les interactions entre nom de 
genre, dispositif énonciatif et visée pragmatique, et évaluant la congruence entre les genres ainsi caractérisés et les formes linguistiques repérables dans les textes (Mellet et Sitri, 2010).

De fait, le monde professionnel se caractérise par l'hétérogénéité des dénominations génériques pour des textes proches dans leurs caractéristiques linguistiques et pragmatiques ${ }^{(4)}$, mais également par l'hétérogénéité des textes regroupés sous la même dénomination générique. C'est le cas également dans le discours universitaire, notamment dans les écrits de recherche « en formation » (Reuter, 2004) : l'initiation à la recherche repose sur des genres diversement nommés (le mémoire, le TER, le rapport de stage, le mémoire professionnel) et le genre du «mémoire » est sans doute moins unifié que sa dénomination peut le laisser croire : il recouvre des attentes diverses suivant les disciplines et que certains auteurs proposent de diversifier davantage encore (par ex. AllinPfister, 2005 ; Perrin, 2005), en regard d'un discours méthodologique jugé normatif et rigide.

Le mail est un bon exemple de cette hétérogénéité, à l'origine de nombreux travaux sur les écrits au travail et les questions de filiation générique dans la communication électronique (voir par ex. Cusin-Berche, 1999 ; Labbé et Marcoccia, 2005 ; Mourlhon-Dalliès, 2007). Dans le même sens, les textes regroupés sous l'appellation de « comptes rendus » peuvent se présenter sous des aspects extrêmement divers. Si l'on compare les exemples réunis en annexes, on relève un compte rendu des débats à l'Assemblée Nationale rédigé par des fonctionnaires indépendants et ayant valeur légale, un compte rendu d'une assemblée telle qu'un comité d'entreprise ou le conseil d'un UFR d'une université, un compte rendu d'une réunion pédagogique rédigé par un des participants et ayant une simple valeur d'enregistrement des décisions prises ou encore un $\mathrm{CR}$ de débats rédigés par des prestataires de service et visant à garder trace du contenu des interventions.

Les différents comptes rendus évoqués ci-dessus se distinguent par le statut du rédacteur (professionnel de la rédaction, secrétaire, participant?), la nature des destinataires (restreint ou tout public ?) ou encore la fonction du compte rendu dans l'institution (simple enregistrement, communication, valeur légale ou non ?). Ces distinctions d'ordre pragmatique et socio-institutionnelles sont alors à corréler avec les variations observées dans les formes prises par les comptes-rendus (fidélité aux propos vs synthèse des discours tenus) et notamment dans le choix des formes de discours rapporté pris dans une acception large de discours autre, et dans leurs degrés de marquage (formes avec autonymie, qu'il s'agisse de discours direct ou d'îlots textuels, formes reformulantes, types de «verbes introducteurs », etc.) (voir en annexes pour des exemples attestant des différences entre compte-rendus).

Ainsi, on pourrait faire l'hypothèse que l'on a affaire à chaque fois à des genres ou sous-genres différents, en tant qu'ils émanent de sphères d'activité distinctes (entreprise, assemblée représentative, université par exemple). Cepen-

(4) Par exemple, le compte-rendu peut s'appeler ainsi ou recevoir des dénominations telles que procès-verbal ou relevé de conclusions. 
dant, en se situant à un niveau de généralité plus grand, on admettra que la visée de tout compte rendu est de garder la trace de ce qui s'est dit dans une assemblée réunie dans un cadre institutionnel - et donc de représenter d'une façon ou d'une autre les dires des participants. Si l'on postule l'existence d'un « genre » ou si l'on veut d'un « macro genre » compte rendu dont la mise en œuvre demande dans tous les cas, du point de vue rédactionnel des compétences analogues de compréhension, de maîtrise du positionnement énonciatif et des procédures de reformulation, on misera alors sur le transfert de compétences comme une clé de la formation (voir Russell dans ce numéro) : l'enjeu est de rendre les étudiants capables de rédiger un compte rendu dans n'importe quelle situation professionnelle. Une des conditions favorisant ce transfert nous semble être de sensibiliser aux différences entre genres et à la diversité des textes regroupés sous une même dénomination générique, selon une approche inspirée de l'analyse de discours et basée sur des enquêtes au sein de diverses sphères d'activité.

\section{Les productions écrites d'étudiants}

L'analyse des textes produits par les étudiants, notre troisième corpus, d'ordre exploratoire, a pour objectif de pointer les compétences rédactionnelles les plus complexes à maîtriser pour ce public ; 1'enjeu est de mettre en place des dispositifs de formation basés sur les besoins effectifs. En nous intéressant à cette question des « compétences rédactionnelles », nous ne renonçons cependant pas à 1 'importance des genres.

Dans le champ des littéracies universitaires, il est désormais établi que la formation à l'écrit doit se faire au travers des genres spécifiques à la discipline enseignée (par ex. Russell, 2002). Le fait de travailler les compétences rédactionnelles à travers des genres disciplinaires permet d'envisager l'écrit en lien avec la construction des savoirs. En se familiarisant avec les genres en usage dans sa discipline, 1'étudiant s'en approprie les savoirs et se familiarise avec des manières de faire et de penser. Par ailleurs, comme les usages de la langue varient avec les genres, ce sont eux qui déterminent les compétences rédactionnelles à mobiliser dans la production d'un texte.

La maîtrise de l'écrit dans le supérieur peut-elle cependant être abordée uniquement et intégralement sous l'angle de la maîtrise des genres disciplinaires? Face à ce qui se présente aujourd'hui comme une revendication récurrente des approches théoriques en didactique et des propositions d'interventions, deux questions nous semblent se poser : d'une part, quelle place faire à et/ou comment travailler la maîtrise de la langue comme maîtrise transversale à différents genres, et répondre ainsi aux besoins des étudiants ne serait-ce qu'en ce qui concerne l'orthographe ou la ponctuation ? D'autre part, en quoi et à quelles conditions les compétences rédactionnelles travaillées via les genres disciplinaires sont-elles transférables à d'autres genres et sphères d'activité ?

La notion de compétences rédactionnelles nous sert à aborder ces questions ; notre hypothèse de travail est qu'en analysant les difficultés rédactionnelles 
dans des genres disciplinaires variés ainsi que dans des genres professionnels, il est possible de mettre en évidence des besoins transversaux, tout autant que la manière dont ils se spécifient en fonction des genres et des contextes. Dans une visée de formation, les compétences clés d'analyse en termes de genres et de leur diversité peuvent être travaillées dans la perspective du contrôle et de la révision des textes, à travers l'observation guidée et des consignes de réécriture.

Nous étudions ici des productions d'étudiants confrontés à la rédaction d'écrits professionnels, dans le cadre d'un master professionnel de sciences du langage accueillant à la fois des étudiants de sciences du langage en formation initiale et des étudiants issus d'autres cursus, en formation continue ou en reconversion. Le cours qui nous a permis de recueillir le corpus est consacré à la pratique de la rédaction selon le principe de «l'étude de cas » : on peut parler également de «mise en situation », en vertu du principe selon lequel la rédaction professionnelle consiste à accomplir un mandat confié par un client ou mandant (Clerc, 2000). Cependant, 1'enjeu est aussi de ne pas s'en tenir à la maîtrise d'une situation spécifique, mais de donner des outils de sensibilisation à la diversité des attentes pour favoriser le transfert des compétences d'une situation à une autre.

Nous prenons ici l'exemple de trois «genres» de textes : le compte rendu, l'affiche et le mail. Comme nous l'avons indiqué plus haut, ces dénominations peuvent correspondre à un « macro-genre » (le CR) ou à un support intégrant différents genres (l'affiche ou le mail). Néanmoins le cadre de l'étude de cas permet d'expliciter le contexte de production des textes et d'en préciser les contraintes énonciatives et pragmatiques, et du coup les propriétés formelles (voir modèle proposé en 3 : il s'agit concrètement de produire le CR exhaustif de débats tenus lors d'une mission d'information à l'Assemblée Nationale, puis une affiche et un mail émanant d'une organisation (l'université en l'occurrence) à destination de l'ensemble de ses membres afin de communiquer une information. Les tâches d'écriture s'inscrivent donc dans le cadre d'un questionnement sur ce que recouvrent les dénominations génériques en termes de normes et de diversité de situations et de textes et la consigne d'écriture cible le genre par une mise en contexte.

Les productions des étudiants font globalement apparaître des difficultés similaires à celles observées en contexte académique, en particulier : la cohérence textuelle, le positionnement énonciatif lié entre autres à la gestion de la représentation des discours autres, 1'expression de la visée pragmatique ou encore la reformulation. Faute de pouvoir tout traiter, nous insisterons dans ce qui suit sur les questions de positionnement du locuteur.

Dans le compte rendu, la difficulté principale est la reformulation. Liée à la compréhension du discours source, elle se traduit dans le genre du CR par la difficulté notamment à saisir la position du locuteur par rapport au thème. On observe également des difficultés à se défaire d'un mode narratif. Cette difficulté a déjà été mise en évidence dans l'écrit académique (Rinck, 2011). Elle est repérable à des marqueurs temporels qui introduisent une « mise en tension » chronologique voire un effet de suspens incompatible avec la visée de 
représentation de dires et à l'absence de marquage explicite d'un point de vue du locuteur, qui sont des propriétés du genre considéré ici.

«C'est alors que Monsieur Jean-Louis Nembrini, directeur général de l'enseignement scolaire au ministère de l'éducation nationale, rebondit sur la question $»$.

Les diverses possibilités de représentation des discours source donnent lieu par ailleurs à des hésitations et, dans les textes, à des alternances entre discours direct et indirect ainsi qu'à un usage abondant de guillemets. On note en outre des maladresses liées à l'inscription de l'énonciateur (« Il nous dit que le fait d'intégrer les IUFM aux universités [...]») et des ambiguïtés dans l'attribution des dires : en particulier les évaluatifs (imputables dans certains cas au scripteur du CR) ou la portée des connecteurs : "Marie-Albane de Suremain demande à répondre car cette question la concerne particulièrement » comme reformulation d'une prise de parole commençant ainsi : «cette question me concerne particulièrement $\gg$.

La rédaction d'une affiche pose des difficultés similaires quant au positionnement du scripteur et à la compréhension du statut de l'écrit au sein de l'institution : contenu non concordant avec le signataire de l'affiche, usage abondant d'un lexique affectif et de points d'exclamation. L'exemple suivant est tiré d'une affiche sur le thème : l'université propose à son personnel et ses étudiants une campagne de vaccination contre la grippe H1N1 :

«Urgent !!! Vaccination contre la grippe H1N1. Les vaccins sont enfin arrivés !!!! La contamination devient inévitable !!! Alors venez nombreux vous faire vacciner (...). Votre santé n'attend pas. "».

Le problème de posture énonciative est clairement lié à des effets d'inadéquation au genre (en termes notamment de proximité et distance) ainsi qu'à la maîtrise de la phraséologie. C'est ce qu'illustre le mail suivant, mail du service entretien destiné aux personnels de l'université :

« Le triage collectif : tous responsables

Madame, monsieur

Nous sommes heureux de vous annoncer que nous avons implanté dans l'enceinte de l'université des poubelles destinées au recyclage. Il est important de rappeler que le recyclage est l'affaire de tous, et que si nous agissons ensemble dès maintenant, la sauvegarde de la planète est possible. Alors n'hésitez pas à vous renseigner sur l'usage de ces containers et de les utiliser dès qu'il le faut.

En vous remerciant de votre attention

L'équipe d'entretien de l'université »

Comme nous l'avons évoqué dans la partie 3., le mail est moins un genre qu'un ensemble de genres hétérogènes et les problèmes posés ici sont pour certains transversaux aux genres (par ex. la compétence lexicale avec triage, n'hésitez pas ...de ) tandis que d'autres sont liés à des effets d'emprunts à d'autres genres (nous sommes heureux de vous annoncer) ; enfin, au niveau pragmatique, l'information délivrée est insuffisante et l'incitation à se renseigner peu convaincante. 
Les constats formulés ici à partir de l'analyse du corpus des écrits professionnels « en formation » (Reuter, 2004) rejoignent les observations faites sur les pratiques des étudiants dans les genres universitaires, notamment sur le discours théorique (lecture et écriture), la prise de notes, le discours autre et le positionnement du scripteur. Ainsi, certaines compétences se présentent comme des compétences clés pour la maîtrise des genres professionnels, de même qu'elles sont centrales dans les genres universitaires, comme celles pointées ici de reformulation, de gestion des sources, de positionnement. Les compétences ciblées sont bien sûr hétérogènes, mais pas seulement d'ordre technique. Il faut sans doute viser non seulement ce qui est le plus sensible à l'évaluation sociale, comme l'orthographe, mais aussi ce qui peut faire prendre conscience du rôle de l'écrit dans la circulation des savoirs - à l'heure du numérique et de la mondialisation des échanges comme souligné dans le champ des New Litteracy Studies (Street, 1993 ; Fraenkel et Mbodj, 2010).

\section{Conclusion}

Nous avons proposé de montrer en quoi la linguistique et les apports récents en analyse de discours peuvent être mis à profit pour la maîtrise de l'écrit professionnel. Les trois corpus que nous avons retenus ont été survolés trop rapidement ici mais permettent de poser quelques jalons en direction d'une formation. Ils font apparaître les besoins en termes rédactionnels, les lacunes dans l'offre existante, et des pistes pour travailler les genres professionnels et les aborder dans leur diversité.

Les objectifs de la formation que nous mettons actuellement en place se structurent - provisoirement peut-être - autour de deux axes : il s'agit d'abord de familiariser les étudiants avec les genres en usage dans diverses sphères professionnelles, de les amener à prendre conscience de la spécificité des genres professionnels par rapport à ceux du monde académique ainsi que de leur variabilité d'un contexte professionnel à un autre, et à s'approprier leurs contraintes énonciatives et pragmatiques. Une formation de ce type peut se concrétiser par un travail de terrain, permettant aux étudiants d'aller à la rencontre de structures et de professionnels : 1'observation participante, la réalisation d'entretiens et la constitution de corpus peuvent les aider à cette recension des genres en usage dans le secteur concerné.

Le second objectif, complémentaire du premier, est de travailler sur les compétences linguistiques transversales et sur la capacité à les mettre en œuvre en fonction des caractéristiques propres à chaque genre. Au sein des différents genres et à partir des productions des étudiants ou de leurs pairs, il s'agit de travailler sur la compétence de contrôle et de révision de textes. Ce travail porte sur différents niveaux du texte (notamment orthographe, syntaxe, reprises anaphoriques, enchaînements thématiques, emploi des connecteurs); 1'accent mis sur l'analyse du discours comme discipline de référence se traduit par une attention spécifique aux phénomènes énonciatifs et pragmatiques.

Il ne s'agit donc pas seulement de former à des modèles de texte (une lettre- 
type ?) ni au bon usage de la langue (que considérer comme une « défaillance» ou une « maladresse » dans un écrit ${ }^{(5)}$ ?). A partir de l'observation de genres divers et de leur mise en contraste, ce travail doit être l'occasion d'un questionnement sur leurs normes et contraintes, sur l'évaluation du lecteur et sur la mâ̂trise par le scripteur des effets que peuvent produire ses textes.

Une formation linguistique aux écrits professionnels a comme limite de ne pouvoir couvrir tous les domaines d'activités en termes de genres et de contenus disciplinaires mais elle propose une méthodologie applicable à différents domaines. Pour autant, elle ne se réduit pas à une approche techniciste des compétences rédactionnelles, car l'analyse de discours impose une contextualisation et appelle à travailler les compétences rédactionnelles à travers les genres effectivement produits dans le monde professionnel.

\section{Références}

Alamargot, D., Terrier, P. \& Cellier, J.-M. (éds) (2005) : Production, compréhension et usages des écrits techniques au travail, Toulouse, Octarès.

ALLIN-PFISTER, A.C.(2005) : Formation à la recherche : une approche globale, Paris, Lamarre.

BAKhtine, M. (1984) : «Les genres de discours », dans Esthétique de la création verbale (texte de 1952-53, publié en 1979), Paris, Gallimard.

BARRÉ DE MinIAC, C. (2003) : «Savoir lire et écrire dans une société donnée », Revue Française de Linguistique Appliquée, 8(1), 107-120.

BARRÉ DE MiniAC, C., BRISSAUD, C. \& RispaiL, M. (eds) (2004) : La littéracie. Conceptions théoriques et pratiques d'enseignement de la lecture-écriture, Paris, L'Harmattan.

BEAUDET, C. (ed) (2001): Recherches en rédaction professionnelle (RRP). Vol. 1-1. [En ligne]. URL : http://pages.usherbrooke.ca/rrp-cb/Beaudet2.pdf

- (2002) : «Lisibilité textuelle et configuration des énumérations dans un texte procédural », Canadian Journal of Applied Linguistics (CJAL)/Revue canadienne de linguistique appliquée (RCLA), 5(1-2), 5-22.

[REICHLER-]BÉGUELIN, M.-J. (1992) : «L'approche des “anomalies” argumentatives », Pratiques, 73, 51-78.

BÉGUELIN, M.-J. (2000) : « Diagnostic des erreurs dans un corpus d'écrits techniques », in La rédaction technique, Bruxelles, De Boeck Université, 105119.

(5) Ou encore comme une « anomalie», pour reprendre les interrogations de M.-J. [Reichler-] Béguelin (1992) à propos des écrits argumentatifs. 
BorzeiX, A. \& Fraenkel, B. (eds) (2001) : Langage et Travail, Communication, Cognition, Action, Paris, CNRS.

Bourgain, D. (1988) : Discours sur l'écriture. Analyse des représentations sociales de l'écriture en milieu professionnel. Thèse de Doctorat d'Etat, Université de Besançon.

— (1990) : «Des représentations sociales de la norme dans l'ordre scriptural », Langue française, 85 (1), 82-101.

BOUTET, J. (2001) : «La part langagière du travail : bilan et évolution », Langage et Société, 98, 17-42.

BRANCA, S. (1999) : «Types, modes et genres : entre langue et discours », Langage et société, 87, 5-24.

Cislaru, G., Katsiki, S., Pugnière, F., Sitri, F., Veniard, M. (2007) : "Quelle continuité entre Analyse du Discours et formation professionnelle : le cas des écrits du signalement d'enfant en danger », in Le français dans le monde Recherches et applications, Le français langue professionnelle : de la compétence linguistique à la maîtrise du poste de travail, 100111.

Clavier, V. \& LAFOnT-Terranova, J. (2005) : «Le français dans une filière technologique : une approche transversale pour l'apprentissage des discours de spécialité », Actes du $9^{\mathrm{e}}$ colloque international de 1'AIRDF, Québec, août 2004, publication sur CD-ROM.

Clerc, I. (2000) : La démarche de rédaction. Québec, Éditions Nota Bene.

ClOT, Y. (1999) : La fonction psychologique du travail, Paris, PUF.

CRINON, J. \& Guigue, M. (2006) : «Écriture et professionnalisation », Revue française de pédagogie, 156, [En ligne] http://rfp.revues.org/621

Cusin-Berche, F. (1999) : «Courriel et genres discursifs », in J. Anis (ed.) Internet, communication et langue française, Paris, Hermès, 31-54.

DABÈNE, M. (1987) : L'adulte et l'écriture. Contribution à une didactique de l'écrit en langue maternelle, Bruxelles, De Boeck.

DelCAMBre, P. (1997) : Ecriture et communications de travail. Pratiques d'écriture des éducateurs spécialisés, Villeneuve d'Ascq, Presses Universitaires du Septentrion.

FILliETTAZ, L. \& BRONCKART, J.-P. (éds.) (2005) : L'analyse des actions et des discours en situation de travail. Concepts, méthodes et applications, Louvain, Peeters.

FrAenKEL, B. et MBODJ, A. (2010) : «Introduction. Les New Literacy studies, jalons historiques et perspectives actuelles », Langage et Société, 133, 724.

KLINKENBERG, J.-M. (2000) : «Introduction à la problématique », in La rédaction technique, Bruxelles, De Boeck Université, 11-24.

LABBÉ, H. \& MARCOCCIA, M. (2005) : «Communication numérique et continuité des genres : l'exemple du courrier électronique », Texto !, 10(3), [En ligne] http://www.revue-texto.net/Inedits/Labbe-Marcoccia.html

LECLERCQ, V. (1999) : Face à l'illettrisme. Enseigner l'écrit à des adultes, Paris, ESF. 
MAingueneAU, D. (2004a) : «Retour sur une catégorie : le genre », in J.-M. Adam, J.-B. Grize et M.A. Bouacha (eds) Texte et discours : catégories pour l'analyse, Dijon, Editions Universitaires de Dijon, 107-118.

- (2004b) : « Typologie des genres institués », (version remaniée des pages 180-187 du Discours littéraire, Paris, A. Colin, 2004, [En ligne] http://pagesperso-orange.fr/dominique.maingueneau/intro_topic.html

Mellet, C. et Sitri, F. (2010) : «Nom de genre et institutionnalisation d'une pratique discursive : le cas de l'interpellation parlementaire et du signalement d'enfant en danger », CMLF 2010, [En ligne] http://www.linguistiquefrancaise.org/ doi 10.1051/cmlf/2010175

MOIRAND, S. (2003) : «Quelles catégories linguistiques pour la mise au jour des genres de discours ", Journée d'étude sur Les genres de l'oral (UMR GRIC-Lyon 2), 18 avril 2003, Actes sur http://gric.univ-lyon 2.fr/Equipe1/actes/journees_genres.htm

MÜNCHOW, VON P. (2007) : «Le genre en linguistique de discours comparative. Stabilités et instabilités séquentielles et énonciatives », Linx, 56, 109-125.

Mourlhon-D Allies, F. (2007) : «Communication électronique et genres de discours », Glottopol, 10. [En ligne] http://www.revue-texto.net/marges/Marges_sommaire.html

OUDART, A.C. (2001): Les chargés de relation clientèle face à la lettre de réclamation, Pratiques, difficultés, Apprentissages, Paris, Presses Universitaires du Septentrion.

- (2002) : «Manuels scolaires et écrits professionnels. Quelle didactique ? », Pratiques 115-116, 181-194.

PERRIN, N. (2004) : « La méthode inductive, un outil pertinent pour une formation par la recherche ? Quelques enjeux pour le mémoire professionnel », Revue des HEP, [En ligne] http://www.revuedeshep.ch/sommaire-2.html

Pery-WoOdley, M.-P. (1993) : Les écrits dans l'apprentissage. Paris, Hachette.

REUTER, Y. (2004) : «Analyser les problèmes de l'écriture de recherche en formation », Pratiques, 121-122, 9-27.

RINCK, F. (2011) : «Former à l'écrit de recherche : quels enjeux, quelles exigences ? » Le Français Aujourd'hui, 174, 79-89.

RusSELL, D. (2002): Writing in the Academic Disciplines : A Curricular Histo$r y$, Carbondale, Southern Illinois University Press, 2nd edition.

STREET, B. V. (1993) : «The new literacy studies », Journal of Research in Reading, 16(2), 81-97,[En ligne] 10.1111/j.1467-9817.1993.tb00039.x

WAgner, D.A., VENEZKY, R.L. et STREeT, B. (eds) (1999) : Literacy. An international handbook. Colorado-Oxford, Westview Press. 


\section{Annexes \\ Le compte-rendu : un genre hétérogène}

Ci-dessous sont réunis les extraits tirés de quatre comptes-rendus. Nous avons souligné quelques formes de marquage des dires autres, pour mettre en évidence les variations existantes entre les types de comptes-rendus.

(1) $\boldsymbol{M} . \boldsymbol{X}$ : «Un de nos vice-présidents a dit que la répercussion sur le plan de charge était inéluctable et que le réétalonnage de la Société était maintenant une réalité que nous confirmera le CCE du 12 Décembre ».

Le Président : «Pour rester réaliste, il faut prendre en compte le fait qu'il n'y ait plus ni fermeture d'usine ni licenciement»

M. Y : «Qui va payer?»

( $\mathrm{PV}$ de réunion plénière de Comité d'établissement)

(2) Mise en place des emplois du temps

Différents membres du Conseil interviennent pour signaler des problèmes qui ne sont pas toujours de détail :

Pour les Lettres modernes, A signale une gestion complexe, en particulier concernant la création des groupes à l'intérieur d'un même cours. B signale que certaines difficultés sont la conséquence d'erreurs portées sur les feuilles d'inscription manuscrites. [...]

(Conseil d'UFR)

(3) On se met d'accord sur les contenus suivants :

1. Relation entre caractéristiques du message et nature du support : en particulier oral/écrit, variation de l'écrit en fonction du support $\rightarrow$ prendre conscience qu'il s'agit de produire un écrit « académique » qui implique prise en compte du lecteur, décentrement par rapport à ses propres productions (important pour le travail sur la réécriture de ses propres productions). 2. Les différents types de textes [...]

(Réunion pédagogique)

(4) Roger Guesnerie approuve le rapport de Jean Tirole sur de nombreux points, qui reprennent d'ailleurs son propre rapport de 2003. La base de l'architecture de Kyoto, soit des quotas par pays, est bonne, en effet, et doit être reprise dans la perspective de Copenhague. Le rapport indique par ailleurs à juste titre que la réduction des émissions de gaz à effet de serre s'accompagne de la recherche de compléments, et non de substituts. [...]

(Séance du Conseil d'Analyse Economique du 9 juin 2009) 